\title{
Joint ordering policy for a conditional trade credit model with two retailers
}

\author{
Zhen Zhang ${ }^{1}$, Songtao Zhang ${ }^{2}$, and Mingshi Yue ${ }^{2}$ \\ ${ }^{1}$ Namseoul University \\ ${ }^{2}$ Linyi University
}

May 5, 2020

\begin{abstract}
This paper focuses on the cooperation mechanism between two retailers. To reduce the average processing cost, the supplier usually sets a threshold for trade credit to stimulate retailers' orders. Retailers can enjoy permissible delay in payments only when their order quantities are more than or equal to the given threshold. However, considering the diversity of retailers, the motivation effect of the threshold may be limited. To resolve the problem, the supplier can additionally provide retailers with a joint ordering policy under which two retailers can make delayed payments as long as their total order quantity meets the required threshold. Thus, the two retailers should decide whether to place a joint order or not and determine their respective order quantities simultaneously. We provide a mutually acceptable order-allocation scheme for retailers, and determine the optimal payment methods for them. In addition, an optimal threshold is identified for the supplier to maximize the total order quantity of retailers. Based on this, some managerial insights are obtained. A numerical experiment is performed to illustrate the validity of the model.
\end{abstract}

\section{Hosted file}

Joint ordering policy for a conditional trade credit model with two retailers.pdf available at https://authorea.com/users/299698/articles/429170-joint-ordering-policy-for-aconditional-trade-credit-model-with-two-retailers 
figures/Figure-1/Figure-1-eps-converted-to.pdf 
figures/Figure-2/Figure-2-eps-converted-to.pdf 
figures/Figure-3/Figure-3-eps-converted-to.pdf 
figures/Figure-4/Figure-4-eps-converted-to.pdf 
figures/Figure-5/Figure-5-eps-converted-to.pdf 
figures/Figure-6/Figure-6-eps-converted-to.pdf 
figures/Figure-7/Figure-7-eps-converted-to.pdf 
figures/Figure-8/Figure-8-eps-converted-to.pdf 
figures/Figure-9/Figure-9-eps-converted-to.pdf 
figures/Figure-10/Figure-10-eps-converted-to.pdf 
figures/Figure-11/Figure-11-eps-converted-to.pdf 